\title{
Cardiovascular Manifestations in Noonan Syndrome: Report of Three Cases
}

\author{
Jesse Jane R. Roque, Felimon K. Morales, Cristine C. Santelices, Pichy Ann P. Alan, \\ Adriano E. Magpali, Jr., Lowe L. Chiong and Paul Ferdinand M. Reganit \\ Section of Cardiology, Department of Medicine, College of Medicine and Philippine General Hospital, University of the Philippines Manila
}

\begin{abstract}
Objectives. Noonan syndrome is a rare disease presenting with phenotypic features. Over two-thirds of patients with Noonan Syndrome have congenital heart defects with pulmonic stenosis as the most common cardiac abnormality. As a means of focusing on these complications, we report three patients with stigmata of Noonan Syndrome, each of whom had a combination of pulmonic stenosis and other cardiac abnormalities.

Patients and Methods. The clinical features and cardiac abnormalities of three patients with Noonan Syndrome were studied, and a literature review on cardiovascular manifestations of this syndrome was undertaken.

Results. The three patients we report had physical features compatible with Noonan Syndrome. Pulmonic stenosis is common among the three cases and other cardiac abnormalities were also noted with the aid of 2D-echocardiogram.

Conclusion. The existence of several types of cardiac abnormalities within one syndrome is unusual and requires further investigation. Thorough history and physical examination is of utmost importance in diagnosing a rare condition with associated cardiac abnormalities. Recognition of common abnormalities in patients with Noonan Syndrome would aid both clinical course and management of this rare condition.
\end{abstract}

Key Words: Noonan Syndrome, pulmonic stenosis, atrial septal defect

\section{Introduction}

Noonan syndrome (NS) is a rare genetic disease presenting with typical morphologic features. It was first \footnotetext{
Hotel, Mandaluyong City.

Corresponding author: Jesse Jane R Roque, MD

Section of Cardiology

Department of Medicine

Philippine General Hospital

University of the Philippines Manila

Taft Avenue, Ermita, Manila 1000 Philippines

Telephone: +632 5548400 local 3670

Email: jroquemd@gmail.com
}

Moderated Poster Presentation, Philippine Heart Association-Philippine College of Cardiology Annual Convention, May 2013, EDSA-Shangrila Plaza recognized by Noonan and Ehmke in 1963 occurring between 1 in 1,000 and 1 in 2,500 live births. ${ }^{1,2}$ Over twothirds of patients with Noonan Syndrome have a congenital heart defect with pulmonic stenosis (PS) as the most common cardiac abnormality. We present three cases of Noonan Syndrome with pulmonic stenosis and other associated cardiac findings.

\section{Case Reports}

\section{Case 1}

A 23-year-old female was referred to our service to seek medical clearance as a prerequisite for employment. On physical examination, patient had phenotypic features of Noonan syndrome which includes short stature, hypertelorism, prominent epicanthal folds, proptosis, prominent outer helix of both ears, webbed neck with prominent neck veins, small mandible, high arched palate, pectus carinatum, and kyphosis (Figure 1A). Apex beat was displaced with a right ventricular heave, 3/6 holosystolic murmur on second to third left intercostal space parasternal area and 2/6 systolic murmur with splitting of S2 on second right intercostal space parasternal area. Chest $\mathrm{x}$-ray showed cardiomegaly with multi-chamber enlargement (Figure 1B). Resting electrocardiogram (ECG) showed sinus rhythm with occasional premature ventricular contractions, right axis deviation (RAD) and right ventricular hypertrophy (RVH) (Figure 1C). Transthoracic and transesophageal echocardiography were done revealing dilated right atrium (RA) and right ventricle (RV) with evidence of pressure and volume overload, dilated left atrium (LA), mitral valve prolapse (MVP), atrial septal defect (ASD), ostium secundum type, dilated coronary sinus, and PS (Figure 2A, 2B, 2C). Hemodynamic study revealed elevated RA, RV, and right ventricular outflow tract (RVOT) systolic pressures. Significant gradient between RVOT and main pulmonary artery (MPA) was noted at $75 \mathrm{mmHg}$ signifying severe PS. An incidental finding of persistent left superior vena cava (PLSVC) draining to the coronary sinus was noted during the study (Figure 2D). Supportive treatment with diuretics, low dose beta blockers and digitalis to ameliorate symptoms of heart failure were given while awaiting surgery to correct ASD and PS. Medications provided improvement in functional capacity. 

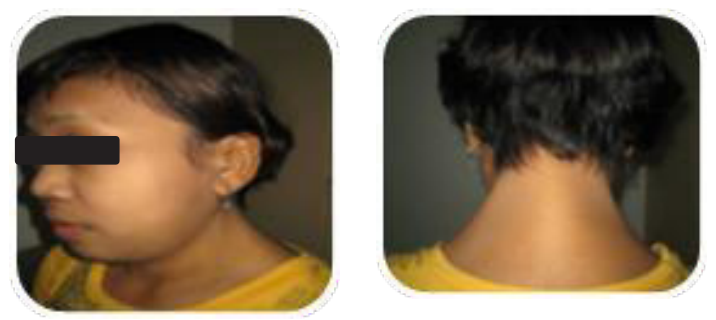

a

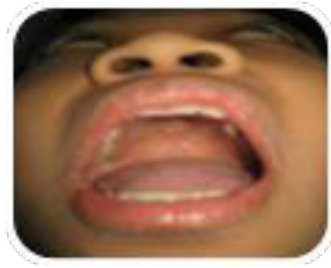

C

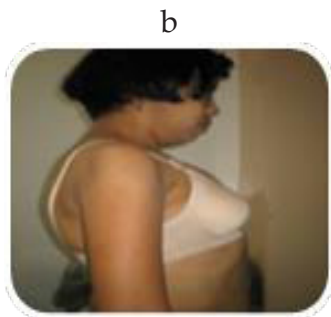

d

A
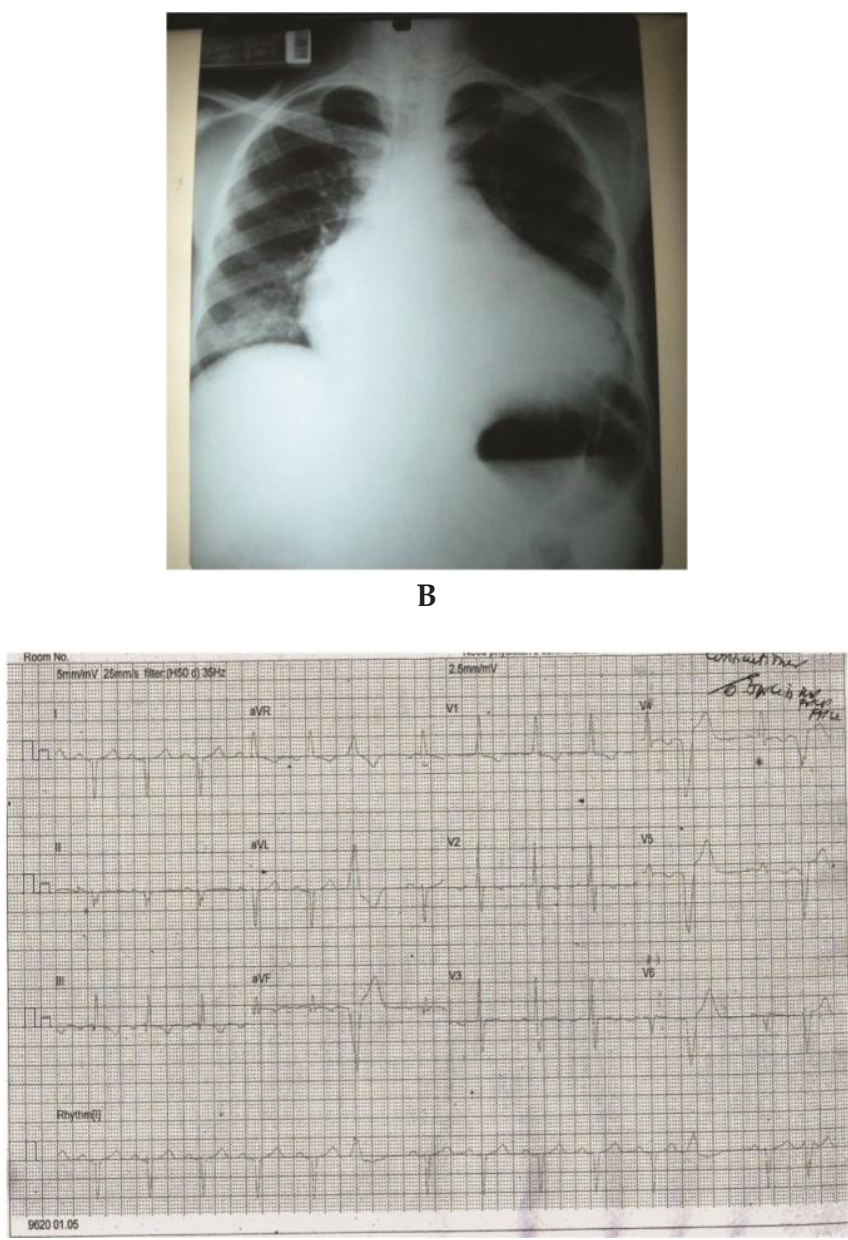

C

Figure 1. Patient 1. (A) Physical features presenting with a) hypertelorism b) webbed neck c) high arched palate d) pectus carinatum and kyphosis. (B) Multichambered cardiomegaly on chest x-ray. (C) ECG showed regular sinus rhythm with occasional premature ventricular contractions, right axis deviation and right ventricular hypertrophy.
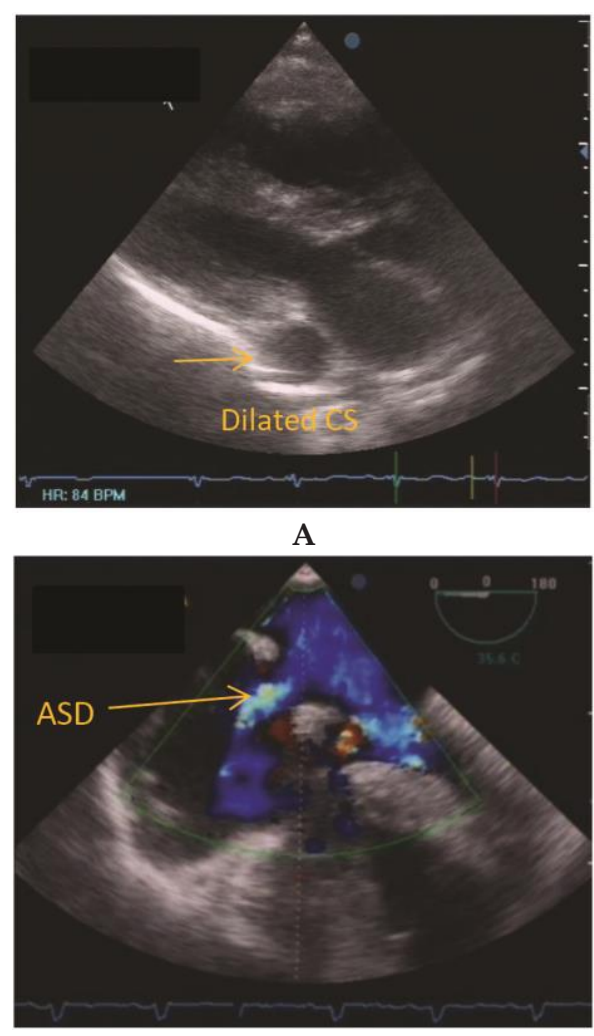

B

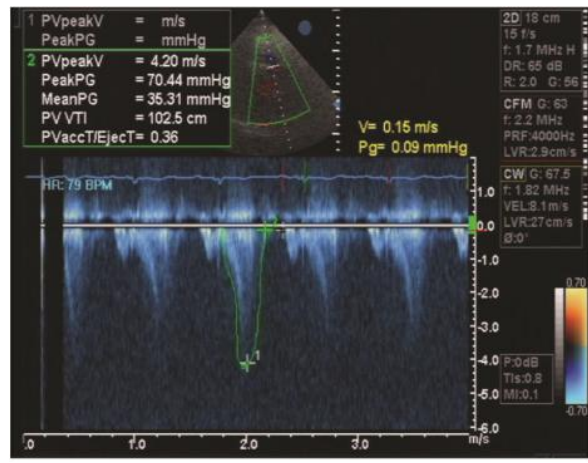

C

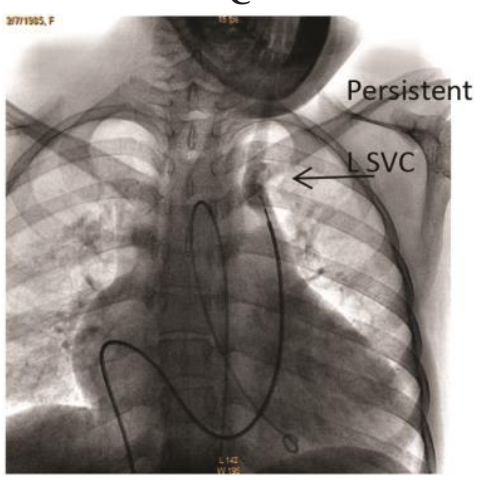

D

Figure 2. Patient 1. (A) transthoracic echo revealing dilated RA, RV and dilated coronary sinus (CS) (B) transesophageal echo showing ASD, Ostium Secundum type. (C) transthoracic echo showing severe PS. (D) Incidental finding of persistent LSVC on hemodynamic study. 


\section{Case 2}

A 19-year-old female was referred for continuation of care from pediatric service. Patient was noted with short stature, broad high forehead, hypertelorism, micrognathia, high arched palate, and webbed neck compatible with NS (Figure 3A). ECG revealed sinus rhythm, extreme RAD, and RVH (Figure 3B). Chest $x$-ray showed no evidence of cardiomegaly (Figure 3C). Transthoracic echocardiogram showed mild PS (peak gradient of $12 \mathrm{mmHg}$ ), ASD, secundum type, dilated RA and dilated RV with no evidence of pressure or volume overload (Figure 4D, 4E). The patient was asymptomatic with no history of failure symptoms hence no medications were given. The patient was advised regular consultation to monitor disease progression.

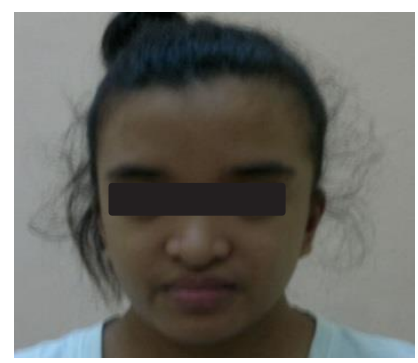

a

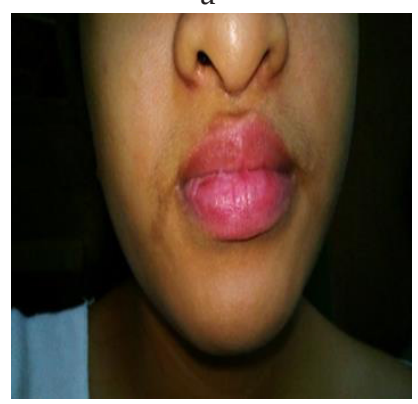

C

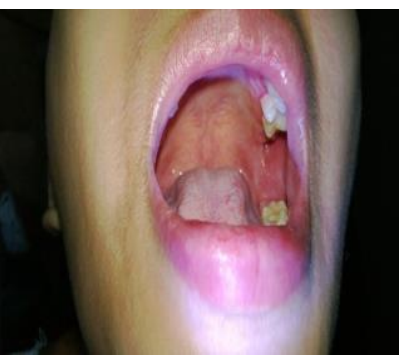

b

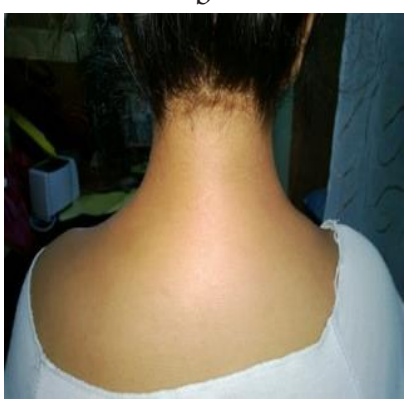

d

A

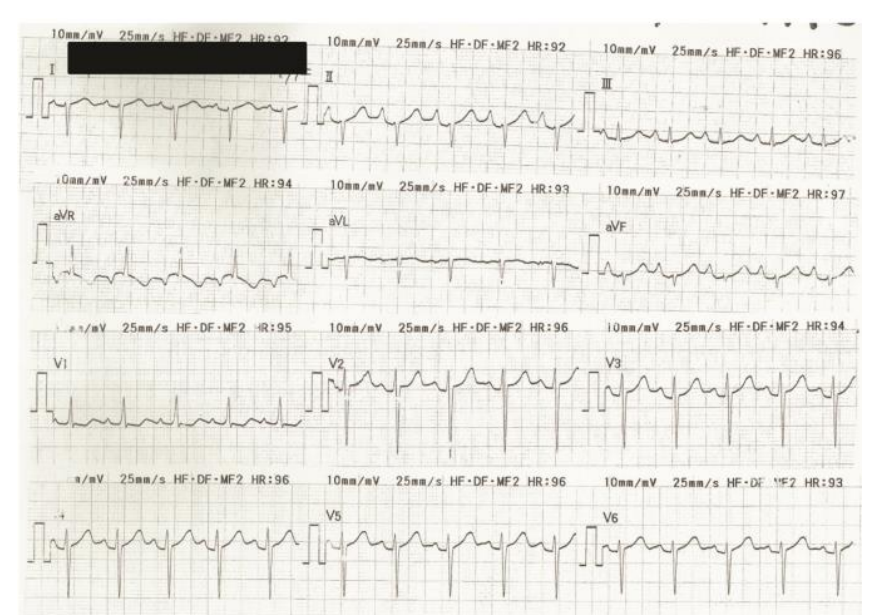

B

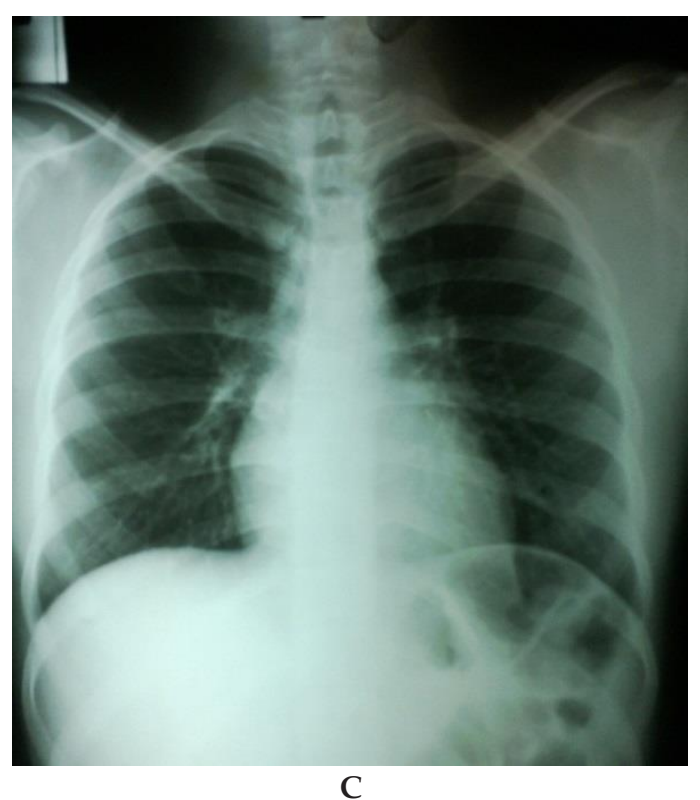

C

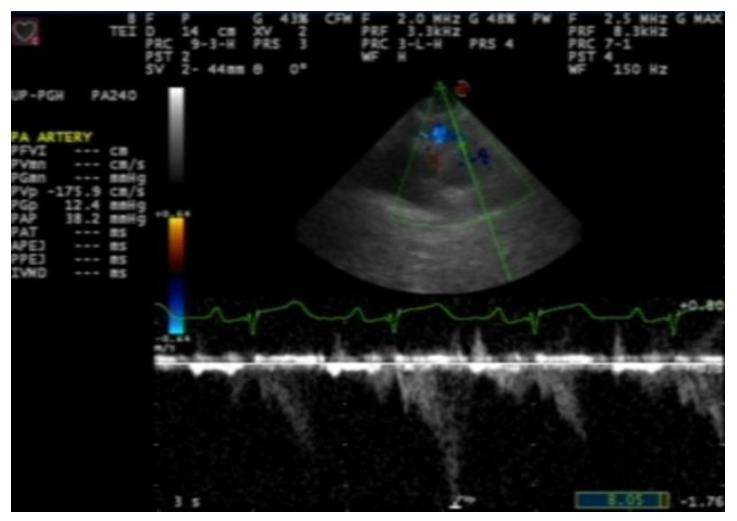

D

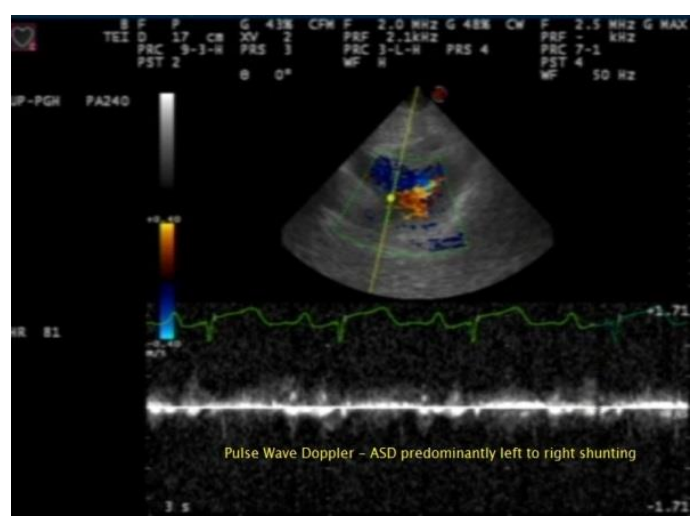

E

Figure 3. Patient 2. (A) Physical features presenting with a) hypertelorism, broad forehead b) high arched palate, c) micrognathia d) webbed neck. (B) ECG showed regular sinus rhythm, right axis deviation and right ventricular hypertrophy. (C) unremarkable chest x-ray. (D) $2 \mathrm{D}$ echocardiogram revealing mild PS and (E)atrial septal defect. 


\section{Case 3}

A 33-year-old female was referred for co-management due to palpitations. On physical examination, patient is obese (BMI $28 \mathrm{~kg} / \mathrm{m}^{2}$ ) with short stature, hypertelorism, high arched palate, and webbed neck (Figure 4A). Cardiac auscultation revealed Grade 5/6 harsh crescendodecrescendo systolic murmur at 2nd intercostal space, left parasternal border suggestive of PS. ECG showed regular sinus rhythm, right atrial abnormality, RAD, and RVH (Figure 4B). Chest $x$-ray showed elongation of right heart border attributed to right atrial enlargement (RAE) and prominent main pulmonary trunk (Figure 4C). 2D Echocardiography revealed severe PS (peak gradient of 89 $\mathrm{mmHg}$ ), normal sized left ventricle with adequate wall motion and contractility and preserved overall systolic function, normal sized LA, dilated RA and RV without signs of pressure and volume overload, mild mitral regurgitation, trivial tricuspid regurgitation, and pulmonic regurgitation (Figure 4D). Physical features and cardiac findings were consistent with NS. Patient complains of occasional easy fatigability and is maintained on propranolol for heart rate control. Surgical intervention as definitive management was advised.

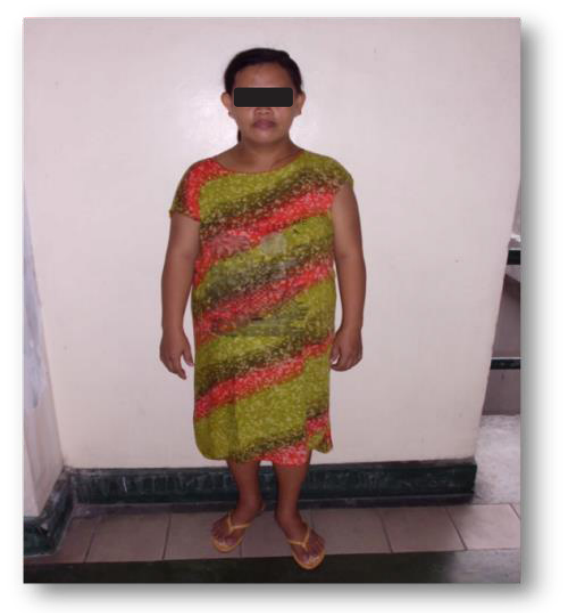

A

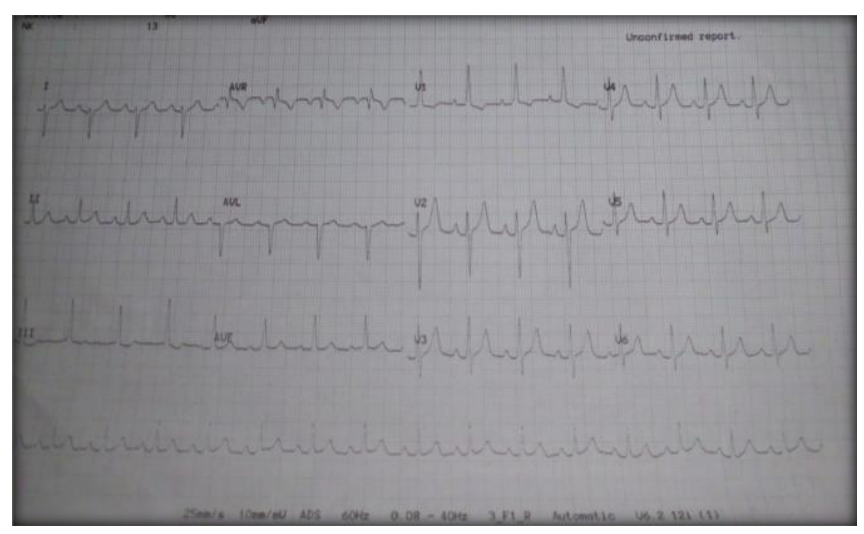

B
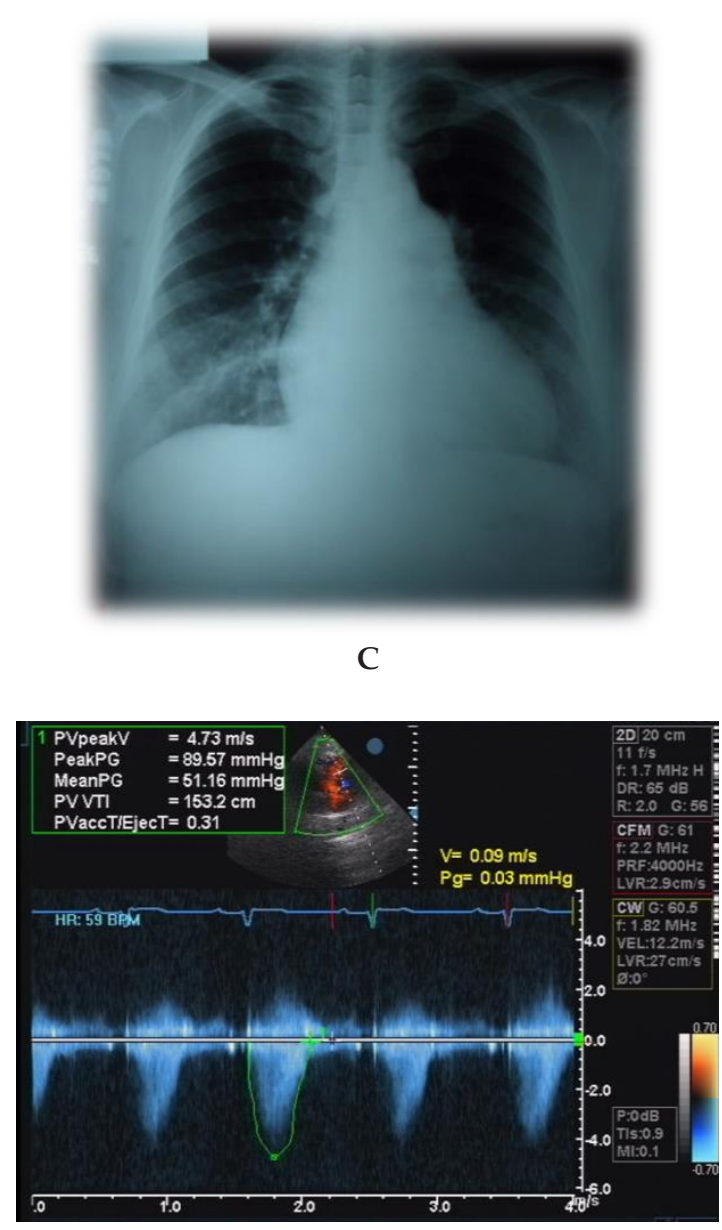

D

Figure 4. Patient 3. (A) Physical features presenting with hypertelorism and webbed neck. (B) ECG showed regular sinus rhythm, right axis deviation and right ventricular hypertrophy. (C)RAE and prominent pulmonary trunk on chest x-ray. (D) 2D echocardiogram revealing severe PS.

\section{Discussion}

Noonan syndrome is a rare autosomal dominant syndrome involving multiple congenital abnormalities: short stature, typical facial dysmorphology (e.g., hypertelorism, epicanthal folds), downward slanting palpebral fissures, ptosis and low-set posteriorly rotated ears with a thickened helix, high arched palate, micrognathia, webbed neck, low posterior hairline, chest deformity, mild intellectual deficit, cryptorchidism, poor feeding in infancy, bleeding tendency, and lymphatic dysplasias. ${ }^{3}$ The physical findings mentioned were seen in the three cases presented hence a diagnosis of Noonan syndrome was made.

Echocardiography remains an important tool in diagnosing cardiac abnormality in Noonan syndrome. In agreement with literature reports, the most frequent cardiac anomaly was pulmonary stenosis, found in most of the patients with cardiac problems. In a study of 45 cases with Noonan Syndrome by Char et al., an interarterial 
communication is the most frequent cardiac defect associated with pulmonary stenosis. ${ }^{7}$ The cardiac finding of pulmonary stenosis in the three cases considerably favors Noonan syndrome. Pulmonary stenosis with dysplastic leaflets is the most frequent congenital heart defect noted in $50-62 \%$ of cases. ${ }^{3}$ Atrial septal defects occur in $6 \%-10 \%$ of cases, ventricular septal defects occur in $5 \%$ of cases and persistent ductus arteriosus occurs in 3\% of cases and structural anomalies of the mitral valve have been reported in the literature. ${ }^{4,5}$ The first two cases presented with atrial septal defect secundum type. Case 2 was asymptomatic while Case 1 presented with symptoms resulting from the effects of long-term left-to-right shunting and subsequent volume loading of the right heart brought about by atrial septal defect. Other cardiac abnormalities in Case 1 include mitral valve prolapse which can contribute to the patient's symptoms and potentially can progress to mitral regurgitation. The presence of a dilated coronary sinus and persistent left superior vena cava are in Case 1, usually a benign condition can predispose the patient to the development of arrhythmia and heart block. ${ }^{6}$

All three cases presented with an electrocardiographic findings of sinus rhythm, right axis deviation, and right ventricular hypertrophy. This is in contrast with the cohort study done by Raaijmakers et al., stating that the electrocardiogram (ECG) of a patient with Noonan syndrome often shows a characteristic pattern, with a left axis deviation, abnormal R/S ratio over the left precordium, and an abnormal Q wave. ${ }^{7}$ This may not be applicable in our patients since the study included pediatric patients as population.

A hemodynamic study can be done to assess severity of valvular stenosis and to adequately evaluate the cardiac lesions found on echocardiography. Only the first case presented underwent hemodynamic study confirming the presence of the previously mentioned cardiac abnormalities (e.g., ASD, PS). An incidental finding of persistent left superior vena cava was also noted draining to the coronary sinus. Incidence of a persistent left superior vena cava (PLSVC) is uncommon. PLSVC is seen in $0.3 \%-0.5 \%$ of the normal population and $1.5 \%-10 \%$ of patients with other congenital heart abnormalities. ${ }^{6}$

Cardiac involvement is common in Noonan syndrome patients and a thorough history and physical examination is of utmost importance in diagnosing this rare condition. Its major morbidity and mortality is dependent on the degree of associated cardiac abnormality. A detailed cardiac evaluation including electrocardiographic and echocardiographic examinations is recommended for every patient with a diagnostic suspicion of Noonan syndrome to properly diagnose, to provide adequate follow up, and to give appropriate management to affected patients.

\section{References}

1. Noonan JA, Ehmke DA. Associated noncardiac malformations in children with congenital heart disease. J Pediatr. 1963; 63:468-70.

2. Allanson JE. Noonan syndrome. J Med Genet. 1987; 24(1):9-13.

3. Allanson JE, Hall JG, Hughes HE, Preus M, Witt RD. Noonan syndrome: the changing phenotype. Am J Med Genet. 1985; 21(3):507-14.

4. Lin AE. Noonan syndrome. J Med Genet. 1988; 25(1):64-5.

5. Sharland M, Burch M, McKenna WM, Patton MA. A clinical study of Noonan syndrome. Arch Dis Child. 1992; 67(2):178-83.

6. Perloff JK. Congenital anomalies of vena caval connection. In: The Clinical Recognition of Congenital Heart Disease, 4th ed. Philadelphia: WB Saunders Company; 1994. pp. 703-14.

7. Char F, Rodriguez-Fernandez HL, Scott CI, Borgaonkar DS, Bell BB, Rowe RD. The Noonan syndrome - a clinical study of forty-five cases. BD: OAS 1972; VIII:110-8. 